\title{
Sparse Representation for Robust 3D Shape Matching
}

\author{
Hong Tu \\ Department of Computer Science \\ Northwest University \\ Shaanxi, China \\ e-mail: 13613841812@139.com
}

\author{
Guohua Geng \\ Department of Computer Science \\ Northwest University \\ Shaanxi, China \\ e-mail: gengguohua@nwu.edu.cn
}

\begin{abstract}
With the number of 3D shapes has risen sharply, a fast and robust matching technology suitable for large 3D shape databases is one of the key technologies to enhance the retrieval performance. We proposed a general novel matching algorithm for 3D shape retrieval: SRRSM, based on sparse representation of signals. Using feature database of 3D shape as over-complete dictionary, the matching problem can be transfer to the problem of sparse representation of signals. It is a second-cone programming (SOCP) problem and can be solved in polynomial time by interior point methods. The proposed approach combines signal reconstruction, sparse and discrimination power in the objective function for matching. It is more sparse and robust for effective matching than the Euclidean distance the most commonly used for matching. Meanwhile, the proposed method is very suitable for large 3D shape database. Theoretical analysis and comparative experiment verify the efficacy of the proposed algorithm.
\end{abstract}

Keywords-sparse representation; matching; $3 D$ shape; robust; large database

\section{INTRODUCTION}

Recently, developments in 3D scanning technologies and hardware-accelerated 3D graphics are making acquisition high quality 3D data easily. As technologies are improving, the number of $3 \mathrm{D}$ models is growing rapidly and a lot of models are already feasible from proprietary and public databases. The problem in creating new 3D models has shifted to search for suitable 3D models in databases or on the Internet. Thereupon, the development of shape matching algorithm is required for the retrieval suitable 3D models from large repositories. The fundamental aim of shape matching is to measure the similarity between two given shapes by employing some distance measure. Therefore, similarity measures are the kernel of every shape matching algorithm.

Since a few years, a large number of articles focus on 3D shape matching. A lot of the current 3D shape matching algorithms are worried on global similarity. Such as [1], who directly analyze the $3 \mathrm{D}$ meshes using curvature correlograms. Some others, such as [2], use a 2D view based method. They offer an analogy nearest neighbor framework to determine the feature views of a 3D model. These methods have rigid transformations invariance for matching similar models. Other methods are enabling retrieval of models different from non-rigid transformations including character articulation or shape bending[3]. They are generally based on the connection graph or skeleton extracting of a 3D model[4][5].

Other approaches to shape matching are met as partially similar shapes are proposed. This kind of shape matching plays a key role in many applications such as indexing or modeling by example. Partial similarity of shape matching plays a central role in a lot of applications such as modeling or indexing by parts. It usually uses the part recognition idea [6]: segmentation the whole shape into significant parts and then matching pairs of parts as whole shapes. Reference [7] also proposed transferring the $3 \mathrm{D}$ shape matching problem to a multi-criterion optimization problem attempting to maximize the similarity and the significance of the matching parts at the same time.

In this paper, we submit a new approach for 3D shape matching based on signal processing: sparse representation for robust shape matching (SRRSM). In our way, the problem of 3D shape matching is transferred into the problem of searching for the sparse representation of signals over an over-complete dictionary. We alter the standard sparse representation framework of signals for 3D shape matching. Firstly, instead of using the generic dictionaries (e.g., Warelet, Fourier, Gabor and Curvelet), we use feature database of 3D shape as an over-complete dictionary whose basic elements are the feature vectors. Secondly, the discrimination power takes the place of the reconstruction error in the objective function of the sparse representation is more appropriate for $3 \mathrm{D}$ shape matching. The proposed approach in this paper combines signal reconstruction, sparse and discrimination power in the objective function for matching. With the theoretical framework of SRRSM, our method is a sparse and more robust for effective matching.

The rest of this paper is organized as follows. Section 2 describes the theory framework of the standard sparse representation of signal. Section 3 discussed the motivations for proposing SRRSM by analyzing the reconstructive methods and discriminative methods for $3 \mathrm{D}$ shape matching. The formulation and solution of SRSM are presented in Section 4. Experimented results with synthetic and real data are showed in Section 5 and Section 6 concludes the paper with a summary of the proposed work and discussions. 


\section{SPARSE REPRESENTATION FOR SIGNAL}

Nowadays, there exists such an ascending momentum in the research field of sparse representations of signals. Sparse representation of signals has applied in many fields including compression, regularization, feature extraction, and more. Using an over-complete dictionary $A \in R^{M \times N}$ that includes prototype signal-atoms as its columns $\left\{d_{j}\right\}_{j=1}^{N}$. A signal $b \in R^{N \times 1}$ can be decomposed as a linear combination of these signal atoms $y=\alpha_{1} d_{1}+\alpha_{2} d_{2}+\ldots \ldots+\alpha_{N} d_{N}$. The signal can be representative as matrix-vector product $y=A x$. The vector $x \in R^{N \times 1}$ contains the representation coefficients $\left(\alpha_{1}, \alpha_{2}, \ldots \ldots, \alpha_{N}\right)$ of the signal $y$. If $M<N$ and $A$ is row full-rank matrix, the solutions of the representation problem ( $y=A x$ ) form a linear subspace. Numerous solutions can available. Hence, constraint functions in the solution will be set for obtaining a unique solution. Classical approach to find an optical solution uses minimize 2-norm:

$$
\left(l^{2}\right): \hat{x}_{2}=\arg \min \|x\|_{2} \text { Subject to } A x=y
$$

( $A \in R^{M \times N}, M<N, x \in R^{N}, b \in R^{M},\|\cdot\|_{2}$ is the 2-norm)

The solution is a minimum-energy solution, having analytically tractable and physical interpretation as minimum energy. But the solution is typically nonzero in every component and the wrong principle for most applications. Therefore the solution with the least nonzero coefficients is absolutely an attractive representation. This sparsest representation can get by solving the following optimization problem:

$$
\left(l^{0}\right): \hat{x}_{0}=\arg \min \|x\|_{0} \text { Subject to } A x=y
$$

( $A \in R^{M \times N}, M<N, x \in R^{N}, b \in R^{M},\|\cdot\|_{0}$ is the 0-norm) In general, solution of (1) requires enumerating all subsets of the direction (matrix $A^{M \times N}$ ) searching the smallest subset can represent the signal $(y)$, and then, the complexity of this algorithm grows exponentially with $N$. It is now know that sparse solution can be obtained by convex optimazation, this has been found empirically $[2,8]$ and theoretically[9-11]. Consider replacing the 0 -norm in (2) by the

1 -norm, getting the minimization 1 -norm problem

$$
\left(l^{1}\right): \arg \min \|x\|_{1} \text { Subject to } A x=y
$$

( $A \in R^{M \times N}, M<N, x \in R^{N}, b \in R^{M},\|\cdot\|_{1}$ is the 1-norm)

This can be viewed as a kind of convexification of (2) [12]. In fact, (3) can be cast as a linear programming problem and solved by modern interior point methods, even for very large number $\mathrm{M}$ and $\mathrm{N}$.

\section{SRRSM THEORY FRAMEWORK}

Sparse representation are important in applications such as de-noising, smoothing and coding, where the original signal $y$ should be reconstructed as precisely as possible. However, for applications like 3D shape matching, the representation coefficients are matching results for the given feature vector. That is to say, the sparest representation is naturally matching: among all feature vectors of feature database, it selects the feature vectors which most compactly expresses the input feature vector and rejects all the others. That is the matching processing.

In this paper, we use sparse representation of signal to perform 3D shape matching. Instead of using the generic dictionaries (e.g. Fourier, Wavelet, Discrete cosine, and Gabor), we use a specifically over-complete dictionary whose column vectors (base elements) are feature vectors of 3D shape feature database. If sufficient feature vectors belong to different classes in feature database are available, the query feature vector can represent as a linear combination of just similar feature vectors. This representation must be sparse, including only little fraction of the overall feature database. In SRRAM, the number of feature vector in database is must enough bigger than dimensionality of feature vector $\left(A^{m \times n}, m<<n\right)$ for building over-complete dictionary. Otherwise feature database can not as over-complete dictionary, and there has not sparse representation for matching.

The SRRSM problem is formulated mathematically as:

$$
\left(l^{1}\right) \min _{x}\|x\|_{1} \text { Subject to } y=A x
$$

Matrix $A \in R^{m \times n}$ is feature database with every column is a $m$-dimision feature vector of a 3D shape. Vector $y \in R^{m \times 1}$ is the feature vector of query 3D shape.

Since the entries of $x$ encode the identity of a 3D shape feature vector $y$, we can find the associated class of $y$ by solving the linear system of equation $y=A x$. In [13], it is proved that if certain conditions on sparse is satisfied, minimize 1-norm will obtain the sparse solution as minimize 0 -norm. Finding solution $x \in R^{n \times 1}$ can be efficiently solved by basis pursuit using linear programming (LP).

When a 3D shape mixing noise as query shape, the matching methods mentioned above may fail because they have little information is included in matching algorithm to deal with noise and missing data. In fact, the feature vector of query 3D shape usually corrupted by noise when conveying on the internet. Therefore, we allow for some degree of noise and solve a noise-aware variant, such as:

$$
\left(l^{1}, \varepsilon\right) \min _{x}\|x\|_{1} \text { Subject to }\|y-A x\|_{2} \leq \varepsilon
$$

The $\varepsilon$ is a relaxation factor,

This is a second-cone programming (SOCP) problem and can be solved in polynomial time by interior point methods. [优化]

\section{EXPERIMENTS AND ANALYSIS}

\section{A. Synthetic Example}

We make the random matrix $A^{20 \times 100}$ as feature database and a random vector $y^{100 \times 1}$ as feature vector of a query $3 \mathrm{D}$ shape. Every random column vector is feature vector of a $3 \mathrm{D}$ shape. The aim is find the most similar feature vector (3D shape descriptor) in the feature database to matching query $3 \mathrm{D}$ shape. If the some columns of matrix $A^{20 \times 100}$ are the most similar to query feature vector, then the corresponding elements of $x$ are nonzero and the other elements are zero. If the $i-t h$ column of $A^{20 \times 100}$ just is the same as query feature vector, then $i-t h$ element of $x$ is one and the other elements are zero. We do synthetic 
experiments to verification the thinks using Euclidean Distance method and SRRSM for matching. The experiment results as follows:

Firstly, the query vector $y=a_{1}$ is one of columns of matri$\mathrm{X} A^{20 \times 100}$. Experimental results as Fig. 1 and Fig. 2. The experiment results show SRRSM has significant high matching effectiveness. It can find the same model while exclude ng those independent models. Euclidean distance metric can also find the same model, but the other independent models have been as return results for matching.

Secondly, the query vector $y\left(y=0.5 a_{7}+0.7 a_{9}+0.3 a_{10}\right)$ is linear combination of some columns in matrix $A^{20 \times 100}$. Experimental results as Fig. 3 and Fig. 4. The experiment $r$ esults show SRRSM has more accurate matching results $\mathrm{f}$ rom Fig. 3. In Fig. 4 we can conclude that Euclidean distance does not return the correct results.

\section{B. Real Example}

We use method in [8] extracting feature vector from one $t$ housand models form feature library and dimensionality o $\mathrm{f}$ feature vector is two hundred and thirty. Extracting query model feature uses the same method. After that, we use SRRSM and Euclidean distance metric as the matching method. The experimental results are as Tab. 1. The Precision-Recall curve is Fig 5. Experimental results show the method has significant high retrieval effectiveness.

\section{CONCLUSION}

The main contribution of this paper is the idea of using sparse representation of signals for robust 3D shape matchin g. The key feature of SRRSM is that it provides a theory framework within matching problems can transfer into a sparse representation of signals, and it is a SOCP problem can be solved in polynomial time by interior point method s. SRRSM includes reconstruction property, discriminateon power and sparsity for robust 3D shape matching. We e-xtensively evaluated the SRRSM on Synthetic and real 3D shape datasets and compared it against Euclidean distance metric method. The evaluation results lead to the conclusion that the SRRSM has very high discriminative power and more robust for 3D shape matching. However, $\mathrm{S}-$ RRSM is suitable for large 3D model database. The number of models in database is much larger than dimensiona- lity of feature vector, so that we can obtain a spare and robust m-atching result. Otherwise, it is not suitable for using SRRSM.

[1] Li, Y., Cichocki, A., \& Amari, S. I,“Analysis of sparse representation and blind source separation". Neural computation,vol.16, 2004, pp.1193-1234,

[2] Pati, Y. C., Rezaiifar, R., \& Krishnaprasad, P. S, "Orthogonal matching pursuit: Recursive function approximation with applications to wavelet decomposition." In Signals, Systems and Computers, 1993. 1993 Conference Record of The TwentySeventh Asilomar Conference on, IEEE. November, 1993,pp.4044.

[3] Chen, S. S., Donoho, D. L., \& Saunders, M. A, "Atomic decomposition by basis pursuit," SIAM journal on scientific computing, vol.20, 1998,pp.33-61.

[4] Donoho D L,"For most large underdetermined systems of linear equations the minimal 1 - norm solution is also the sparsest solution,"Communications on pure and applied mathematics, vol.59,2006,pp. 797-829.

[5] Yan R, Shao L, Liu Y, "Nonlocal hierachical dictionary learning using wavelets for image denoising", IEEE transactions on image processing,vol.22, Dec.2013, pp.4689-4698.

[6] Chen Y, Nasrabadi N M, Tran T D. Hyperspectral image classification via kernel sparse representation[J]. Geoscience and Remote Sensing, IEEE Transactions on, vol.51,2013, pp.217-231.

[7] Chen S S, Donoho D L, Saunders M A, "Atomic decomposition by basis pursuit", SIAM journal on scientific computing, vol.20, 1998,pp. 33-61.

[8] Osada R, Funkhouser T, Chazelle B, et al, "Matching 3D models with shape distributions", Shape Modeling and Applications, SMI 2001 International Conference on. IEEE, 2001,pp.154-166.

[9] Donoho D L, Huo X, IEEE Translate information theory,vol.47,2001, pp.2845-2862.

[10] Elad M, Bruck stein A M, In processings of the IEEE international conference on image processing (IEEE, New York),2001.

[11] Elad M, Bruck stein A M, In processings of the IEEE international conference on image processing (IEEE, New York), vol.48, 2001, pp.2558-2567.

[12] Donoho, David L., and Michael Elad. "Optimally sparse representation in general (nonorthogonal) dictionaries via $\ell 1$ minimization." Proceedings of the National Academy of Sciences, vol.100, 2003, pp.2197-2202.

[13] Lee K, Bresler Y, "Admira: Atomic decomposition for minimum rank approximation", Information Theory, IEEE Transactions on, vol. 56, 2010,pp.4402-4416.

[14] Boyd S P, Vandenberghe L. Convex optimization[M]. Cambridge university press, 2004 


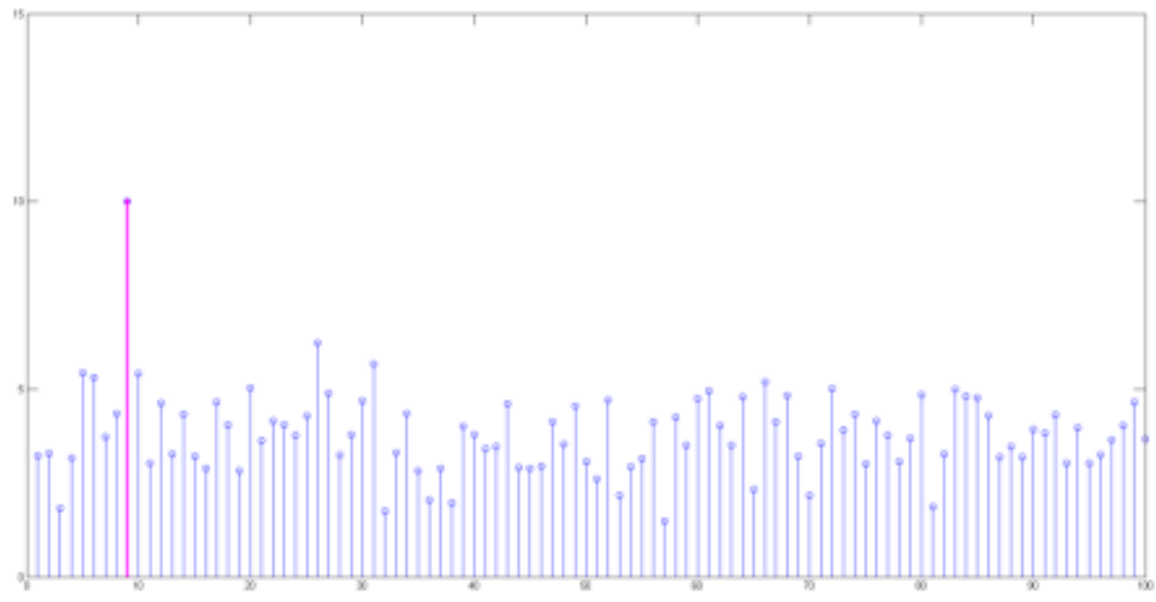

Figure 1. Euclidean Distance as metric

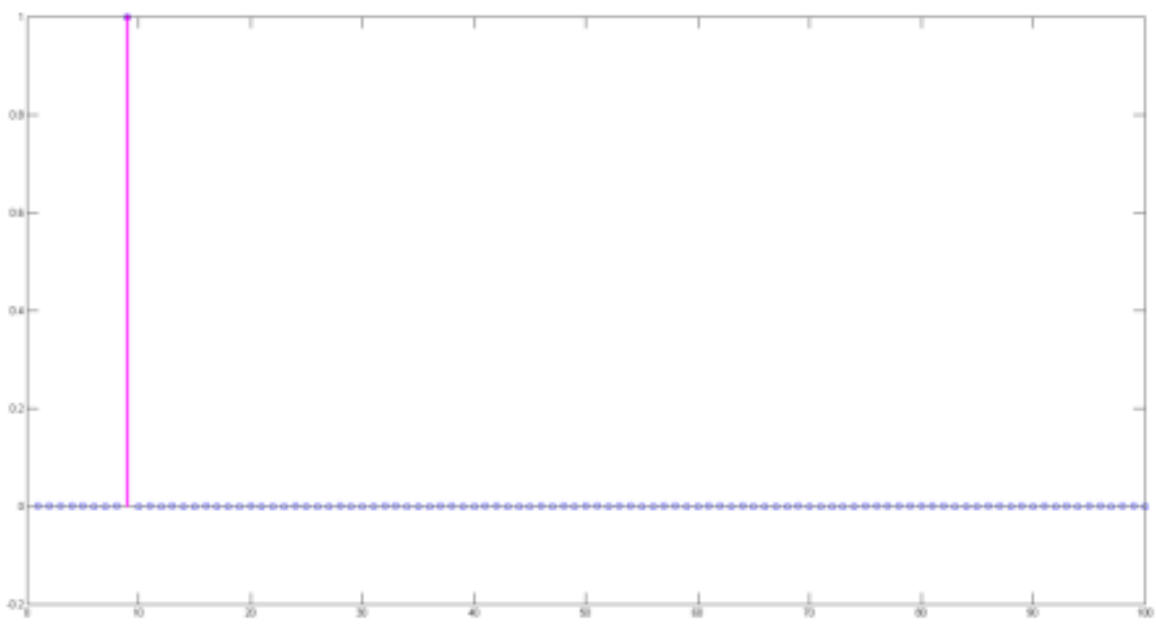

Figure 2. SRRSM method

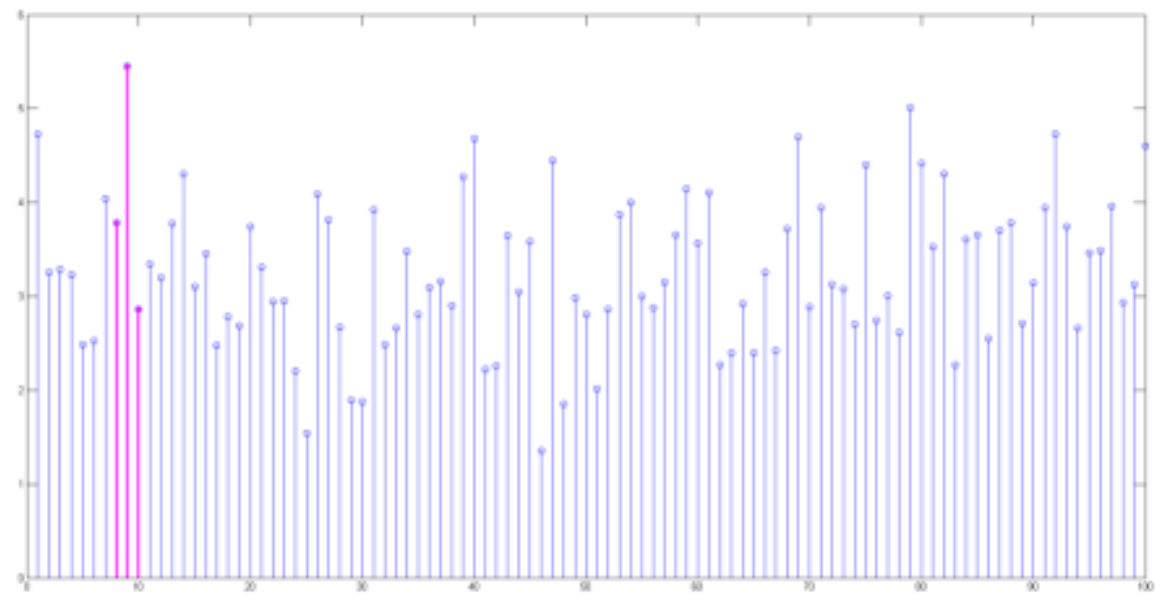

Figure 3. Euclidean Distance as metric 


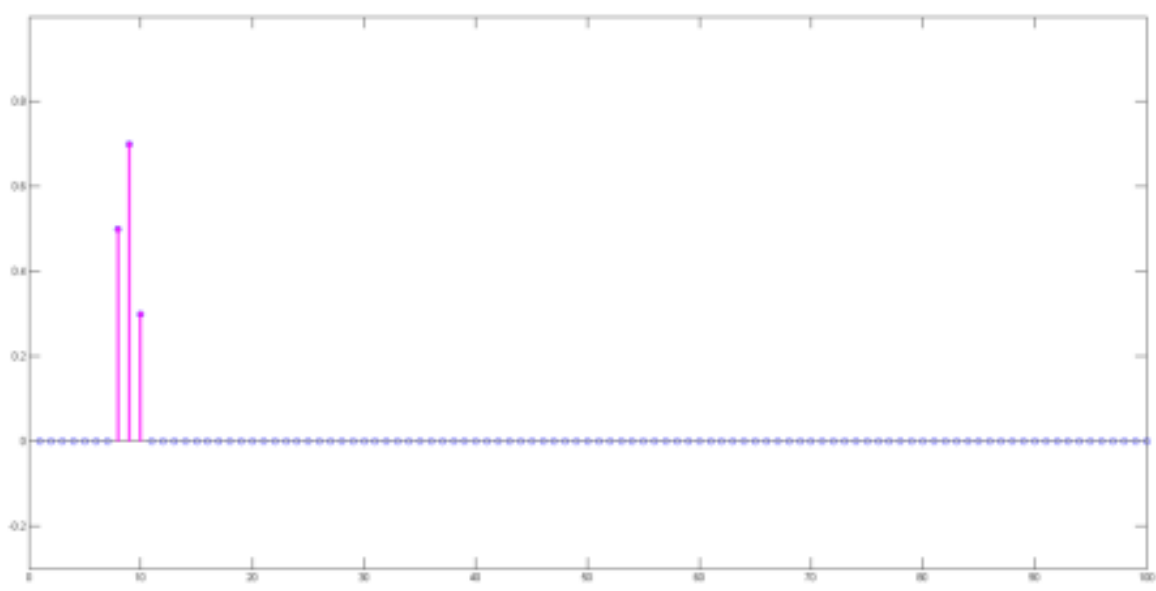

Figure 4. SRRSM method

TABLE 1. Some experiment results

\begin{tabular}{|c|c|c|c|c|c|c|}
\hline $\begin{array}{c}\text { Matching } \\
\text { method }\end{array}$ & Input model & Result_1 & Result_2 & Result_3 & Result_4 & Result_5 \\
\hline SRRSM & & & & & & \\
\hline $\begin{array}{c}\text { Euclidean } \\
\text { Distance }\end{array}$ & & & & & & \\
\hline SRRSM & & & & & & \\
\hline Euclidean & & & & & & \\
\hline & & & & & & \\
\hline
\end{tabular}

